\title{
Endoscopic surveillance or ablation for Barrett's esophagus?
}

\author{
John M. Inadomi, Nina Saxena \\ Division of Gastroenterology, Department of Medicine, University of Washington School of Medicine, Seattle, WA 98195, USA \\ Contributions: (I) Conception and design: All authors; (II) Administrative support: JM Inadomi; (III) Provision of study materials or patients: All \\ authors; (IV) Collection and assembly of data: All authors; (V) Data analysis and interpretation: All authors; (VI) Manuscript writing: All authors; \\ (VII) Final approval of manuscript: All authors. \\ Correspondence to: John M. Inadomi, MD. Division of Gastroenterology, Department of Medicine, University of Washington School of Medicine, 1959 \\ NE Pacific Street, Box 356424, Seattle, WA 98195, USA. Email: jinadomi@medicine.washington.edu.
}

\begin{abstract}
The incidence of esophageal adenocarcinoma (EAC) is rising and the only known precursor of this disease is Barrett's esophagus (BE). EAC mortality remains high, prompting strategies to screen individuals with gastroesophageal reflux disease (GERD) symptoms to identify BE and conduct surveillance in order to detect neoplasia at a stage that is amenable to cure. The effectiveness of endoscopic eradication therapy has been improving with reduced harms, yet it is unclear which patients will benefit from this procedure. This chapter reviews the evidence supporting surveillance for BE to reduce mortality from EAC and combines these results with economic analyses to identify the optimal means to manage patients with BE with high-grade dysplasia, low-grade dysplasia, or no dysplasia.
\end{abstract}

Keywords: Mass screening; endoscopy; surveillance; cost-effectiveness; Barrett's esophagus (BE); esophageal adenocarcinoma (EAC)

Received: 30 July 2018; Accepted: 28 September 2018; Published: 06 December 2018.

doi: $10.21037 / \operatorname{tgh} .2018 .11 .09$

View this article at: http://dx.doi.org/10.21037/tgh.2018.11.09

\section{Introduction}

Survival of patients with symptomatic esophageal adenocarcinoma (EAC) is poor $(1,2)$. Strategies that prevent cancer by eradicating Barrett's esophagus (BE) with dysplasia, or detect cancer at an early, curable stage have been implemented to reduce cancer mortality. Screening is the process by which asymptomatic populations are tested to identify individuals who have early-stage cancer or a pre-malignant condition that predisposes them to develop cancer. High-risk individuals identified through screening either undergo treatment of cancer (or the pre-malignant condition) or surveillance, in which testing is repeated to identify disease at a curable stage. Guidelines from national societies recommend screening in selected populations and surveillance for those who have BE; however, there is controversy about who to screen, what tests to use, how to perform surveillance and when to treat $(3,4)$.

This chapter summarizes the results of two independently conducted systematic reviews of the published literature.
One review retrieved English language publications of human research and clinical studies in PubMed from August 2001 through August 2016 with MESH search terms (Barrett's esophagus, esophageal adenocarcinoma, screening, surveillance, endoscopy) to identify studies that examine whether endoscopy reduces mortality from EAC (5). A total of 8,405 publications were identified by this search and 51 reported the incidence or mortality from EAC. After exclusion of studies that did not have a comparison group of individuals not undergoing endoscopic surveillance and studies lacking data on mortality from EAC, 14 studies were included in this review. None of the studies were prospective and the majority relied on administrative data that did not differentiate between a screening and surveillance endoscopy; thus, "surveillance" is used describe any upper endoscopy prior to the diagnosis of cancer.

A second systematic review summarized in this chapter added a meta-analysis and expanded the search period to include 1996-2017, using search terms such as esophageal 
adenocarcinoma, Barrett esophagus, dysplasia, and endoscopic surveillance to query MEDLINE, Cochrane CENTRAL, SCOPUS, Web of Science, PubMed and Ovid EMBASE (6). Of 1,747 records identified through this query, 1,631 were excluded due to lack of relevance to the study question $[1,419]$ or format of a narrative review or editorial [212]. The remaining 116 publications were screened and an additional 97 studies were excluded due to the lack of: inclusion of the outcome of interest [50], appropriate comparison group [44], data for the comparison group [1], or human subjects [2]. Of the 116 full text articles reviewed, 19 met all inclusion and exclusion criteria for this meta-analysis.

This chapter further excludes from discussion studies from either systematic review for which the benefit of endoscopy on EAC mortality could not be ascertained due to the absence of the predictor or outcome variables, or the combination of endpoints (such as combining high-grade dysplasia and cancer). Combined, these systematic reviews describe 16 studies for which cancer mortality can be compared between individuals undergoing screening and/ or surveillance endoscopy with individuals not receiving endoscopy (Table 1).

\section{The effectiveness of endoscopic screening and surveillance to reduce EAC mortality}

Due to the barriers based on the size and duration needed to conduct a properly powered randomized controlled trial, the evidence supporting the use of screening and surveillance to reduce mortality from EAC cancer is limited to case-control or cohort studies. A typical case-control study compares patients who died of cancer with controls who either did not have cancer or did not die of cancer. After ascertaining the outcome of each patient in the study (i.e., whether they died of EAC), investigators examine records to determine which patients underwent screening or surveillance endoscopy prior to the cancer diagnosis. However, in a case-control study the prevalence of cancers is set by the study design and is a function of the ratio of cases to controls. Because of this, a case-control study does not calculate the cancer mortality reduction associated with screening (i.e., the risk ratio). Instead, case-control studies provide an "odds ratio", which is the odds that a patient who died of cancer underwent a prior endoscopy compared with the odds that a patient who did not have cancer (or had cancer but did not die of cancer) had a prior endoscopy. It is reassuring, though, that for diseases with a low incidence rate (e.g., $1 \%$ or lower as is the case with esophageal cancer) the odds ratio (OR) approximates the risk ratio and is a good estimate of the cancer mortality reduction associated with endoscopic screening and surveillance.

A cohort study, which can be prospective or retrospective, is another study design that can estimate the effectiveness of endoscopic screening and surveillance on cancer mortality. This type of study follows a group of patients with $\mathrm{BE}$ (cohort) over time to determine the incidence and mortality from cancer. Individuals who receive endoscopic screening and surveillance are compared with those who do not receive endoscopy to calculate the hazard ratio, or the incidence of cancer in patients who receive endoscopy divided by the incidence in those who do not receive endoscopy. The advantage of the cohort study compared with a case-control study is that the incidence of cancer is observed in a cohort study, allowing the reduction in cancer risk to be calculated with endoscopic screening and surveillance. The barriers to a cohort study include identifying an adequately sized cohort, following patients long enough to observe cancer development, and incomplete data due to drop-out of patients from the cohort.

Non-randomized studies have a higher risk of bias than randomized controlled trials. Selection bias occurs because individuals who undergo endoscopy are usually healthier and therefore more likely to live longer than individuals who do not undergo endoscopy. Lead-time bias may also be present, which represents a longer observed survival with screening due to detection of pre-clinical cancer. Lengthtime bias can occur when there is heterogeneity in the natural history of cancer. Slowly growing cancers are more likely to be detected since they span several surveillance intervals compared with rapidly growing cancers that may arise between surveillance intervals. This creates the appearance that endoscopic surveillance prolongs survival when the truth is that cancers diagnosed by surveillance are more indolent. Statistical techniques are employed to mitigate the effects of bias, but it is difficult to identify and adjust for all sources of bias in non-randomized studies.

\section{The effectiveness of endoscopic surveillance}

Early outcomes studies in the 1990's and early 2000's using a retrospective cohort study design reported that endoscopic surveillance could reduce EAC mortality. Patients with EAC who had undergone endoscopic surveillance had significantly better survival than patients who had not 
Table 1 Endoscopic screening and surveillance to reduce mortality from esophageal adenocarcinoma

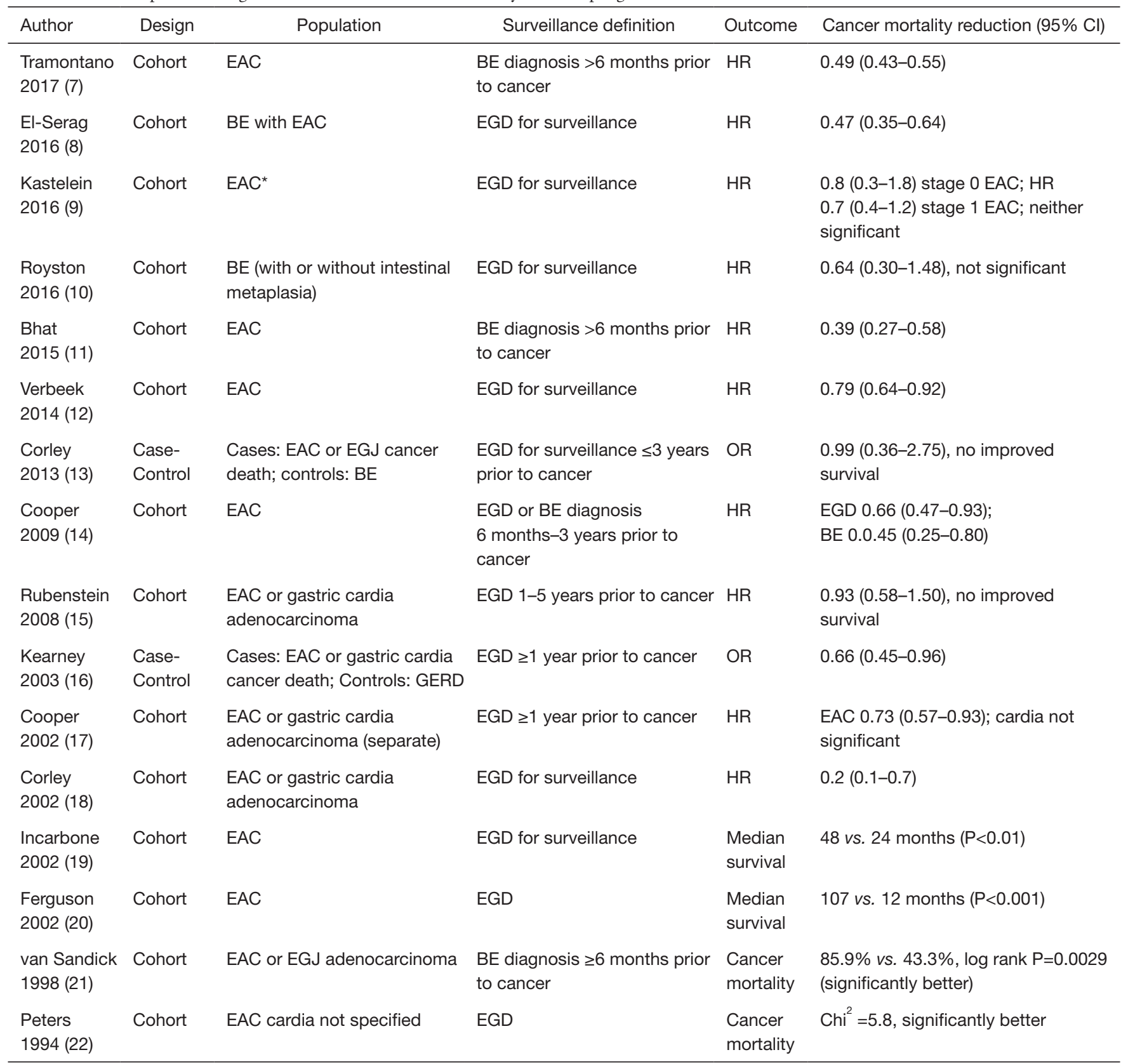

*, comparison between observed and expected survival. EAC, esophageal adenocarcinoma; EGJ, esophago-gastric junction; GERD, gastroesophageal reflux disease; BE, Barrett's esophagus; EGD, esophagogastroduodenoscopy; HR, hazards ratio; OR, odds ratio; Cl, confidence interval.

undergone endoscopy (19-22). These studies, however, used limited statistical techniques that could not adjust for the potential biases of the retrospective design.

In a retrospective cohort study using more advanced statistical methods, Cooper et al. reported that patients who had an upper endoscopy at least one year prior to the cancer diagnosis had $27 \%$ lower cancer mortality than patients with EAC who did not have endoscopy [hazards ratio (HR), 0.73; 95\% confidence interval (CI): 0.57-0.93] (17). Using a different data set but similar study design, Corley and others reported an $80 \%$ reduction in cancer death in patients with EAC who had undergone endoscopic surveillance compared 
with no surveillance (18). Kearney et al. reported that individuals with symptoms of gastroesophageal reflux who had received an upper endoscopy greater than one year prior to the diagnosis of EAC patients had a 34\% lower incidence of cancer mortality (OR, 0.66; 95\% CI: 0.45-0.96) (16).

The indication for upper endoscopy was not known in these studies since administrative or billing data were used to identify endoscopy use. Thus, it is not clear that all upper endoscopies were performed for surveillance since they could have been performed in response to symptoms or signs related to esophageal cancer. To address this limitation, Cooper and colleagues conducted a cohort study of patients with EAC, using the diagnosis of BE 6 months to 3 years prior to development of EAC as a surrogate for surveillance endoscopy (14). Using this surrogate could reduce the risk that endoscopy was performed for persistent reflux symptoms that would identify a group of individuals at higher risk of cancer, or for symptoms of cancer itself. They found that individuals diagnosed with $\mathrm{BE}$ prior to developing cancer were $55 \%$ less like to die of cancer compared with patients who did not have a prior diagnosis of BE.

Using retrospective cohort designs, subsequent studies have demonstrated prolonged cancer survival among patients who were undergoing endoscopic surveillance. Verbeek reported a $21 \%$ reduction in death from cancer among patients with BE who underwent endoscopic surveillance compared with those who did not undergo endoscopy (12). Similar to the Cooper study of the benefit of a prior diagnosis of $\mathrm{BE}$, Bhat and others reported that having a diagnosis of $\mathrm{BE}$ before receiving a diagnosis of cancer was associated with $61 \%$ reduction in cancer death (HR, 0.39; 95\% CI: 0.27-0.58) (11). El-Serag published a cohort study of patients with BE who developed EAC during a mean follow up of five years (8). Patients in whom endoscopic surveillance was conducted, identified through manual chart abstraction, had lower cancer mortality compared with patients who did not undergo surveillance endoscopy $(34 \%$ vs. $54 \% ; \mathrm{P}<0.0001)$. A significant benefit of surveillance endoscopy on cancer mortality was demonstrated even after adjustment for age, race, comorbidity, year of cancer diagnosis, number of clinic visits, and the propensity to undergo upper endoscopy with a HR of 0.47 (95\% CI: $0.35-0.64)$. The benefit of surveillance was largely explained by diagnosis at a lower stage of cancer plus the increased likelihood of cancer treatment among patients who received surveillance.

However, there are additional studies that have not demonstrated a benefit from endoscopic surveillance. Kastelein reported that compared with national cancer statistics there was no difference in cancer death among a cohort of patients with BE undergoing surveillance (9). In a follow-up to their earlier cohort study, Corley et al. published a study comparing patients with BE who died of EAC with controls with BE who did not die of EAC (13). In contrast to their prior findings, they reported that endoscopy within three years of a diagnosis of EAC did not prolong survival. While patients with EAC were less likely to have undergone prior endoscopy, this finding was not statistically significant.

Rubenstein also reported no improvement in survival among patients receiving endoscopy in a retrospective cohort study of patients with EAC (HR, 0.93; 95\% CI: $0.58-1.50)(15)$. A unique aspect of this study was a seven-year follow-up period after the cancer diagnosis. A secondary analysis limiting the follow up to five years found a significant mortality benefit from endoscopy, suggesting that lead-time bias could be a problem with studies using a shorter follow-up.

Tramontano et al. identified almost 5,000 patients with EAC in linked SEER-Medicare data to determine whether a diagnosis of $\mathrm{BE}$ as proxy for endoscopic screening and surveillance was associated with reduced cancer mortality (7). After adjusting for potential confounders (age, sex, race, year of cancer diagnosis, geographic region, marital status, income, education level, comorbidities, and treatment), the hazards of cancer-related death was reduced among patients with a prior diagnosis of BE (HR, 0.49; 95\% CI: $0.43-0.55)$. Inclusion of cancer stage and type of treatment in the hazards model should eliminate the mortality benefit since these are the two plausible mechanisms through which cancer mortality could be reduced with endoscopic screening and surveillance; however, despite inclusion of these factors the benefit of a prior Barrett's diagnosis remained, suggesting residual confounding. Further analysis incorporating correction for lead-time bias reduced the observed mortality benefit to non-significant values (HR, 0.89; 95\% CI: 0.78-1.01). Their conclusion was that patients with EAC with a prior diagnosis of BE have better overall- and cancer-specific survival compared with cancer patients who do not have a prior diagnosis of $\mathrm{BE}$ and presumably do not undergo screening or surveillance. However, their detailed analysis revealed that much of the observed benefit could be a result of lead and/or length time bias, reducing our confidence that screening and surveillance reduced cancer mortality. 
The difference in outcomes reported between studies may be explained by the limitations in their study design. The best study to determine the effectiveness of endoscopic screening to decrease mortality of EAC would be performed in a group of people who did not know whether they had BE: these individuals would be randomized to undergo endoscopy or no endoscopy. Endoscopy would identify patients with $\mathrm{BE}$ who would undergo surveillance endoscopy at intervals based on the presence or absence of dysplasia, or other markers of increased cancer risk. All patients would be followed for a duration sufficient to observe cancer incidence and mortality. Patients with dysplasia or early cancer would undergo endoscopic eradication therapy and esophagectomy would be performed in patients with malignancy extending beyond the mucosa. The primary outcome of this study would be a comparison of cancer mortality between individuals randomized to screening with individuals randomized to no screening. Secondary endpoints would include cancer incidence, stage at the time of diagnosis, and overall mortality between the two groups.

There is an ongoing multicenter clinical trial in the United Kingdom of 3,400 patients diagnosed with BE randomized to endoscopic surveillance every 2 years, or non-surveillance (or "at need" endoscopy in response to clinical symptoms or signs). While the benefit of screening to detect BE will remain elusive, this powerful study is expected to provide evidence to support or refute the benefits of endoscopic surveillance among individuals diagnosed with $\mathrm{BE}$ on esophageal cancer incidence and mortality (23).

An important consideration of the discussed studies was that they used data collected prior to the widespread use of endoscopic radiofrequency ablation (RFA) (24-26). Photodynamic therapy (PDT) may have been available, but its use was limited $(27,28)$. The role of surveillance prior to the availability of endoscopic eradication therapy was to diagnose cancer at a stage amenable to cure. In contrast, current endoscopic eradication therapy aims to treat cancer precursors such as dysplasia with the aim of reducing cancer incidence. Future cohort studies may determine the effectiveness of surveillance when endoscopic eradication is used to treat neoplasia and early stage cancer.

\section{Cost-effectiveness of endoscopic surveillance in patients with BE}

Other chapters in this review will cover the effectiveness of endoscopic eradication therapy for patients with $\mathrm{BE}$ and low- or high-grade dysplasia. The focus of this chapter is on the benefit of surveillance, limiting the scope of this discussion to the point where surveillance identifies a lesion that should trigger treatment. Since there are no clinical studies available to answer this question, we rely on other means to estimate the relative benefits of surveillance versus therapy for patients who develop a treatable lesion. One quantitative tool available to compare different options for medical management is medical decision analysis. A specialized version of decision analysis is a cost-effectiveness analysis (CEA) that allows comparison of the benefits of competing strategies in relation to the resources needed to implement the strategies (29-31).

This chapter summarizes the results of a systematic review of English language publications in PubMed conducted from August 2001 through August 2016 using MESH terms including BE; esophageal neoplasms diagnosis; health care economics and organizations. Studies were included if they reported both the costs and the effectiveness of endoscopic surveillance and used metrics of life-years or quality-adjusted life-years gained.

\section{Surveillance versus endoscopic therapy for high grade dysplasia (HGD)}

Due to the high rate of stricture after PDT, endoscopic mucosal resection for nodular dysplasia followed by RFA of flat mucosa has become the preferred method of endoscopic treatment for BE with HGD. This strategy is associated with fewer complications than esophagectomy, is highly effective in eradicating dysplasia and metaplasia and has a low rate of dysplasia recurrence $(3,4)$. Six studies reported the cost-effectiveness of RFA for HGD compared with endoscopic surveillance with esophagectomy for cancer (Table 2) (32-37). In all studies, RFA with or without endoscopic mucosal resection yielded more quality-adjusted life-years gained at a lower cost than surveillance with esophagectomy for cancer.

\section{Surveillance versus endoscopic eradication therapy for low grade dysplasia (LGD)}

Optimal management of patients with $\mathrm{BE}$ and low-grade dysplasia is controversial (Table 3). Analyses conducted prior to the advent of endoscopic eradication therapy found that endoscopic surveillance for LGD was cost-effective compared with no surveillance or esophagectomy; however, 
Table 2 Cost-effectiveness of surveillance or endoscopic eradication in Barrett's esophagus with high-grade dysplasia

\begin{tabular}{|c|c|c|c|}
\hline Author/year & Population & Strategies & Most cost-effective strategy \\
\hline \multirow{2}{*}{ Hu 2016 (32) } & & Esophagectomy & \\
\hline & & RFA & \\
\hline \multirow[t]{2}{*}{ Kastelein 2015 (33) } & 55-year-old men with HGD & None & RFA \\
\hline & & Esophagectomy & \\
\hline \multirow[t]{2}{*}{ Hur 2012 (34) } & 50-year-old patients with HGD & Surveillance & RFA \\
\hline & & RFA & \\
\hline Boger 2010 (35) & 64-year-old men with HGD & Esophagectomy & RFA \\
\hline Pohl 2009 (36) & & Esophagectomy & \\
\hline \multirow[t]{6}{*}{ Inadomi 2009 (37) } & 50-year-old patients with HGD & None & RFA \\
\hline & & RFA with surveillance & \\
\hline & & APC with surveillance & \\
\hline & & PDT with surveillance & \\
\hline & & Surveillance & \\
\hline & & Esophagectomy & \\
\hline
\end{tabular}

HGD, high grade dysplasia; RFA, radiofrequency ablation; ND, non-dysplastic; BE, Barrett's esophagus; LGD, low grade dysplasia; APC, argon plasma coagulation; PDT, photodynamic therapy.

the optimal surveillance interval was unclear $(40,41)$. Kastelein $e t$ al. analyzed a variety of surveillance intervals for BE and LGD, with patients undergoing endoscopic eradication therapy for a diagnosis of HGD. Surveillance endoscopy every 3 years among patients with BE and LGD was cost-effective ( $€ 32,000$ per QALY gained); however, annual surveillance was also within the willingness-topay threshold. Similarly, Gordon et al. estimated that surveillance of non-dysplastic BE every three years for nondysplastic Barrett's and annually for patients with LGD was cost-effective (38).

In a more recent analysis using updated data of the effectiveness of endoscopic eradication therapy, Hur et al. calculated that endoscopic mucosal resection for nodular lesions followed by RFA for LGD was cost-effective compared with surveillance in LGD, with an incremental cost-effectiveness ratio of $\$ 18,200$ per quality-adjusted lifeyear gained. The optimal strategy, however, depended on the rate of progression from LGD to EAC incorporated into the model, and the amount that society was willing to pay per quality adjusted life year gained (34). Clinical practice guidelines from national societies differ in management recommendations for patients with $\mathrm{BE}$ and LGD, recommending either surveillance every 6-12 months or endoscopic eradication therapy $(3,4)$.

\section{Endoscopic ablation of non-dysplastic BE}

Endoscopic eradication therapy for non-dysplastic BE is not a cost-effective strategy. The most recent economic analysis by Hur et al. reported that endoscopic eradication therapy for patients with $\mathrm{BE}$ without dysplasia costs between $\$ 118,000$ and $\$ 205,000$ per QALY gained compared with surveillance, reserving therapy for patients who develop low- or high-grade dysplasia (34). For the foreseeable future, endoscopic surveillance of non-dysplastic BE with endoscopic ablation for dysplasia remains a more costeffective strategy (Table 3). 
Table 3 Cost-effectiveness of surveillance or endoscopic eradication for Barrett's esophagus with low-grade dysplasia or no dysplasia

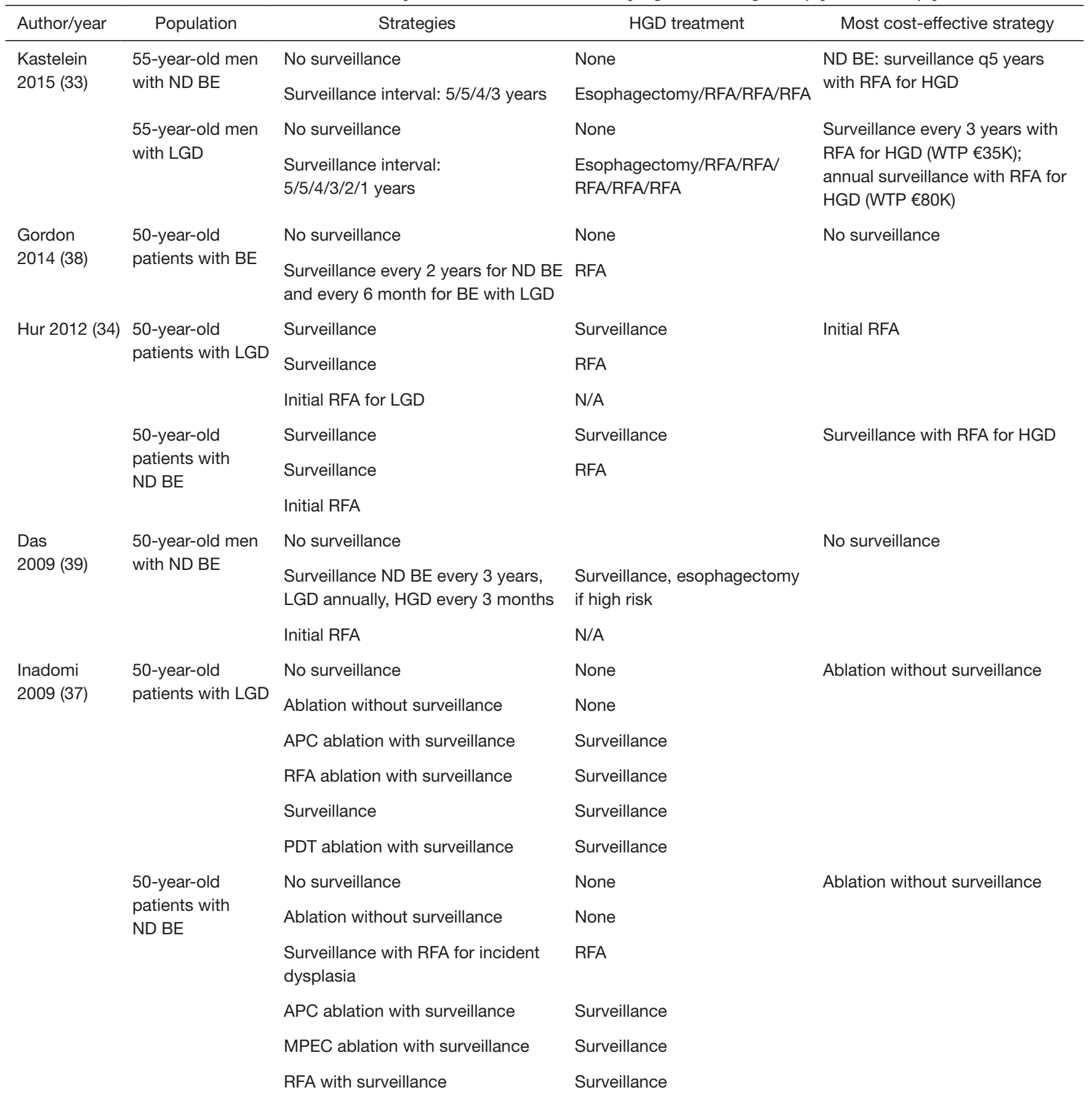

ND, non-dysplastic; BE, Barrett's esophagus; RFA, radiofrequency ablation; HGD, high grade dysplasia; LGD, low grade dysplasia; APC, argon plasma coagulation; MPEC, multipolar electrocoagulation.

\section{Non-endoscopic screening for BE}

Expansion of screening for $\mathrm{BE}$ will be expensive unless nonendoscopic tests are used. Non-sedated, office-based testing is possible with the Cytosponge ${ }^{\mathrm{TM}}$, an ingestible capsule comprised of compressed mesh attached to a string. The capsule is swallowed and the mesh expands in the stomach, obtaining cytologic sampling of the esophagus when the 
string is withdrawn. The cells obtained in this manner are tested for the presence of trefoil factor 3, which is an immunohistochemical marker that has been demonstrated to have a sensitivity of $73.35 \%$ and specificity of $93.8 \%$ for BE (42). An economic analysis conducted by Benaglia et al. demonstrated that Cytosponge ${ }^{\mathrm{TM}}$ screening for $\mathrm{BE}$ with endoscopic mucosal resection and RFA for patients diagnosed with HGD is a cost-effective strategy. The costeffectiveness of this approach is $\$ 15,000$ per QALY gained compared with no screening, and is most cost-effective than screening with standard endoscopy (43).

\section{Summary}

Strategies to reduce mortality from EAC are evolving. We have an increasing understanding of the natural history of $\mathrm{BE}$ and its transformation to EAC, and our ability to stratify cancer risk is improving. The endoscopic treatment of dysplasia is also improving with increased effectiveness and reduced harms. The economic analyses discussed in this review unambiguously support endoscopic eradication therapy for patients with BE with HGD. Unfortunately, the management of LGD is not as straightforward due to the histopathological difficulty in the diagnosis of LGD and disparate estimates of the cancer risk. Patients with non-dysplastic BE are optimally managed with endoscopic surveillance and future efforts should be aimed at identifying the sub-group of non-dysplastic patients who possess an elevated risk of cancer and would benefit from endoscopic eradication.

\section{Acknowledgements}

None.

\section{Footnote}

Conflicts of Interest: The authors have no conflicts of interest to declare.

\section{References}

1. El-Serag HB, Sweet S, Winchester CC, et al. Update on the epidemiology of gastro-oesophageal reflux disease: a systematic review. Gut 2014;63:871-80.

2. Hur C, Miller M, Kong CY, et al. Trends in esophageal adenocarcinoma incidence and mortality. Cancer
2013;119:1149-58.

3. Shaheen NJ, Falk GW, Iyer PG, et al. ACG Clinical Guideline: Diagnosis and Management of Barrett's Esophagus. Am J Gastroenterol 2016;111:30-50.

4. Spechler SJ, Sharma P, Souza RF, et al. American Gastroenterological Association medical position statement on the management of Barrett's esophagus. Gastroenterology 2011;140:1084-91.

5. Saxena N, Inadomi JM. Effectiveness and CostEffectiveness of Endoscopic Screening and Surveillance. Gastrointest Endosc Clin N Am 2017;27:397-421.

6. Codipilly DC, Chandar AK, Singh S, et al. The Effect of Endoscopic Surveillance in Patients With Barrett's Esophagus: A Systematic Review and Meta-analysis. Gastroenterology 2018;154:2068-86.e5.

7. Tramontano AC, Sheehan DF, Yeh JM, et al. The Impact of a Prior Diagnosis of Barrett's Esophagus on Esophageal Adenocarcinoma Survival. Am J Gastroenterol 2017;112:1256-64.

8. El-Serag HB, Naik AD, Duan Z, et al. Surveillance endoscopy is associated with improved outcomes of oesophageal adenocarcinoma detected in patients with Barrett's oesophagus. Gut 2016;65:1252-60.

9. Kastelein F, van Olphen SH, Steyerberg EW, et al. Impact of surveillance for Barrett's oesophagus on tumour stage and survival of patients with neoplastic progression. Gut 2016;65:548-54.

10. Royston C, Caygill C, Charlett A, et al. The evolution and outcome of surveillance of Barrett's oesophagus over four decades in a UK District General Hospital. Eur J Gastroenterol Hepatol 2016;28:1365-73.

11. Bhat SK, McManus DT, Coleman HG, et al. Oesophageal adenocarcinoma and prior diagnosis of Barrett's oesophagus: a population-based study. Gut 2015;64:20-5.

12. Verbeek RE, Leenders M, Ten Kate FJ, et al. Surveillance of Barrett's esophagus and mortality from esophageal adenocarcinoma: a population-based cohort study. Am J Gastroenterol 2014;109:1215-22.

13. Corley DA, Mehtani K, Quesenberry C, et al. Impact of endoscopic surveillance on mortality from Barrett's esophagus-associated esophageal adenocarcinomas. Gastroenterology 2013;145:312-9.e1.

14. Cooper GS, Kou TD, Chak A. Receipt of previous diagnoses and endoscopy and outcome from esophageal adenocarcinoma: a population-based study with temporal trends. Am J Gastroenterol 2009;104:1356-62.

15. Rubenstein JH, Sonnenberg A, Davis J, et al. Effect 
of a prior endoscopy on outcomes of esophageal adenocarcinoma among United States veterans. Gastrointest Endosc 2008;68:849-55.

16. Kearney DJ, Crump C, Maynard C, et al. A case-control study of endoscopy and mortality from adenocarcinoma of the esophagus or gastric cardia in persons with GERD. Gastrointest Endosc 2003;57:823-9.

17. Cooper GS, Yuan Z, Chak A, et al. Association of prediagnosis endoscopy with stage and survival in adenocarcinoma of the esophagus and gastric cardia. Cancer 2002;95:32-8.

18. Corley DA, Levin TR, Habel LA, et al. Surveillance and survival in Barrett's adenocarcinomas: a population-based study. Gastroenterology 2002;122:633-40.

19. Incarbone R, Bonavina L, Saino G, et al. Outcome of esophageal adenocarcinoma detected during endoscopic biopsy surveillance for Barrett's esophagus. Surg Endosc 2002;16:263-6.

20. Ferguson MK, Durkin A. Long-term survival after esophagectomy for Barrett's adenocarcinoma in endoscopically surveyed and nonsurveyed patients. J Gastrointest Surg 2002;6:29-35.

21. van Sandick JW, van Lanschot JJ, Kuiken BW, et al. Impact of endoscopic biopsy surveillance of Barrett's oesophagus on pathological stage and clinical outcome of Barrett's carcinoma. Gut 1998;43:216-22.

22. Peters JH, Clark GW, Ireland AP, et al. Outcome of adenocarcinoma arising in Barrett's esophagus in endoscopically surveyed and nonsurveyed patients. J Thorac Cardiovasc Surg 1994;108:813-21.

23. Old O, Moayyedi P, Love S, et al. Barrett's Oesophagus Surveillance versus endoscopy at need Study (BOSS): protocol and analysis plan for a multicentre randomized controlled trial. J Med Screen 2015;22:158-64.

24. Shaheen NJ, Sharma P, Overholt BF, et al. Radiofrequency ablation in Barrett's esophagus with dysplasia. N Engl J Med 2009;360:2277-88.

25. Fleischer DE, Overholt BF, Sharma VK, et al. Endoscopic radiofrequency ablation for Barrett's esophagus: 5-year outcomes from a prospective multicenter trial. Endoscopy 2010;42:781-9.

26. Shaheen NJ, Overholt BF, Sampliner RE, et al. Durability of radiofrequency ablation in Barrett's esophagus with dysplasia. Gastroenterology 2011;141:460-8.

27. Overholt BF, Panjehpour M, Halberg DL. Photodynamic therapy for Barrett's esophagus with dysplasia and/or early stage carcinoma: long-term results. Gastrointest Endosc 2003;58:183-8.
28. Overholt BF, Lightdale CJ, Wang KK, et al. Photodynamic therapy with porfimer sodium for ablation of high-grade dysplasia in Barrett's esophagus: international, partially blinded, randomized phase III trial. Gastrointest Endosc 2005;62:488-98.

29. Russell LB, Gold MR, Siegel JE, et al. The role of costeffectiveness analysis in health and medicine. Panel on Cost-Effectiveness in Health and Medicine. JAMA 1996;276:1172-7.

30. Weinstein MC, Siegel JE, Gold MR, et al. Recommendations of the Panel on Cost-effectiveness in Health and Medicine. JAMA 1996;276:1253-8.

31. Siegel JE, Weinstein MC, Russell LB, et al. Recommendations for reporting cost-effectiveness analyses. Panel on Cost-Effectiveness in Health and Medicine. JAMA 1996;276:1339-41.

32. Hu Y, Puri V, Shami VM, et al. Comparative Effectiveness of Esophagectomy Versus Endoscopic Treatment for Esophageal High-grade Dysplasia. Ann Surg 2016;263:719-26.

33. Kastelein F, van Olphen S, Steyerberg EW, et al. Surveillance in patients with long-segment Barrett's oesophagus: a cost-effectiveness analysis. Gut 2015;64:864-71.

34. Hur C, Choi SE, Rubenstein JH, et al. The cost effectiveness of radiofrequency ablation for Barrett's esophagus. Gastroenterology 2012;143:567-75.

35. Boger PC, Turner D, Roderick P, et al. A UK-based cost-utility analysis of radiofrequency ablation or oesophagectomy for the management of high-grade dysplasia in Barrett's oesophagus. Aliment Pharmacol Ther 2010;32:1332-42.

36. Pohl H, Sonnenberg A, Strobel S, et al. Endoscopic versus surgical therapy for early cancer in Barrett's esophagus: a decision analysis. Gastrointest Endosc 2009;70:623-31.

37. Inadomi JM, Somsouk M, Madanick RD, et al. A costutility analysis of ablative therapy for Barrett's esophagus. Gastroenterology 2009;136:2101-14.

38. Gordon LG, Mayne GC, Hirst NG, et al. Costeffectiveness of endoscopic surveillance of non-dysplastic Barrett's esophagus. Gastrointest Endosc 2014;79:242-56.

39. Das A, Wells C, Kim HJ, et al. An economic analysis of endoscopic ablative therapy for management of nondysplastic Barrett's esophagus. Endoscopy 2009;41:400-8.

40. Gerson LB, Groeneveld PW, Triadafilopoulos G. Cost-effectiveness model of endoscopic screening and surveillance in patients with gastroesophageal reflux 
disease. Clin Gastroenterol Hepatol 2004;2:868-79.

41. Inadomi JM, Sampliner R, Lagergren J, et al. Screening and surveillance for Barrett esophagus in high-risk groups: a cost-utility analysis. Ann Intern Med 2003;138:176-86.

42. Kadri SR, Lao-Sirieix P, O'Donovan M, et al. Acceptability and accuracy of a non-endoscopic screening test for

doi: $10.21037 / \operatorname{tgh} .2018 .11 .09$

Cite this article as: Inadomi JM, Saxena N. Endoscopic surveillance or ablation for Barrett's esophagus? Transl Gastroenterol Hepatol 2018;3:102.
Barrett's oesophagus in primary care: cohort study. BMJ 2010;341:c4372.

43. Benaglia T, Sharples LD, Fitzgerald RC, et al. Health benefits and cost effectiveness of endoscopic and nonendoscopic cytosponge screening for Barrett's esophagus. Gastroenterology 2013;144:62-73. 